\title{
ACTION OF HECKE OPERATORS \\ ON SIEGEL THETA SERIES I
}

\author{
LyNNE H. WALLing
}

\begin{abstract}
Aвstract. We apply the Hecke operators $T(p)$ and $\widetilde{T}_{j}\left(p^{2}\right)(1 \leq j \leq n, p$ prime $)$ to a degree $n$ theta series attached to a rank $2 k \mathbb{Z}$-lattice $L, n \leq k$, equipped with a positive definite quadratic form in the case that $L / p L$ is hyperbolic. We show that the image of the theta series under these Hecke operators can be realized as a sum of theta series attached to certain closely related lattices, thereby generalizing the Eichler Commutation Relation (similar to some work of Freitag and of Yoshida). We then show that the average theta series (averaging over isometry classes in a given genus) is an eigenform for these operators. We show the eigenvalue for $T(p)$ is $\epsilon(k-n, n)$, and the eigenvalue for $T_{j}^{\prime}\left(p^{2}\right)$ (a specific linear combination of $T_{0}\left(p^{2}\right), \ldots, T_{j}\left(p^{2}\right)$ ) is $p^{j(k-n)+j(j-1) / 2} \beta(n, j) \epsilon(k-j, j)$ where $\beta(*, *), \epsilon(*, *)$ are elementary functions (defined below).
\end{abstract}

\section{INTRODUCTION AND STATEMENTS OF RESULTS}

Let $L$ be a rank $2 k \mathbb{Z}$-lattice $\left(k \in \mathbb{Z}_{+}\right)$equipped with a positive definite quadratic form $Q$. So $L=\mathbb{Z} v_{1} \oplus \cdots \oplus \mathbb{Z} v_{2 k}$ and with $B$ the symmetric bilinear form associated to $Q$ so that $Q(v)=B(v, v), A=\left(B\left(v_{i}, v_{j}\right)\right)$ is a symmetric matrix with

$$
Q\left(a_{1} v_{1}+\cdots+a_{2 k} v_{2 k}\right)=\left(\begin{array}{lll}
a_{1} & \cdots & a_{2 k}
\end{array}\right) A\left(\begin{array}{c}
a_{1} \\
\vdots \\
a_{2 k}
\end{array}\right) .
$$

By scaling $Q$ if necessary, we can assume $Q(L) \subseteq 2 \mathbb{Z}$ (so $L$ is even integral). Set

$$
\theta(L ; \tau)=\sum_{C \in \mathbb{Z}^{2 k, n}} e\left\{{ }^{t} C A C \tau\right\}
$$

where $\tau \in \mathcal{H}_{n}$ (so $\tau=X+i Y, X, Y$ real, symmetric $n \times n$ matrices with $Y>0$ ) and $e\{*\}=\exp (\pi i \operatorname{Tr}(*))$. Then $\theta(L ; \tau)$ is a Siegel modular form of weight $k$, degree $n$,

1991 Mathematics Subject Classification. 11F41.

Key words and phrases. Siegel Modular Forms, Theta Series, Hecke Operators.

Typeset by $\mathcal{A}_{\mathcal{M}} \mathcal{S}-\mathrm{T}_{\mathrm{E}} \mathrm{X}$ 
level $N$ and character $\chi$ where $N$ is the smallest positive integer so that $N A^{-1}$ is an even integral matrix, and for primes $p$ not dividing $N$,

$$
\chi(p)= \begin{cases}1 & \text { if } L / p L \text { is hyperbolic } \\ -1 & \text { otherwise }\end{cases}
$$

(see [1]). Also, one sees that

$$
\theta(L ; \tau)=\sum_{T} r(A, T) e\{T \tau\}
$$

where $T$ varies over all symmetric positive semi-definite even integral $n \times n$ matrices, and

$$
r(A, T)=\#\left\{C \in \mathbb{Z}^{2 k, n}:{ }^{t} C A C=T\right\} .
$$

So $\left(v_{1}, \ldots, v_{2 k}\right) C$ varies over all $\left(x_{1}, \ldots, x_{n}\right), x_{i} \in L$. Let $\Lambda$ be the (formal) direct sum $\mathbb{Z} x_{1} \oplus \cdots \oplus \mathbb{Z} x_{n}$ equipped with the (possibly semi-definite) quadratic form given by $T=\left(B\left(x_{i}, x_{j}\right)\right)$. Let

$$
e\{\Lambda \tau\}=\sum_{G} e\{T[G] \tau\}
$$

where $G$ varies over $G L_{n}(\mathbb{Z})$ (or, if $k$ is odd, we equip $\Lambda$ with an orientation and let $G$ vary over $S L_{n}(\mathbb{Z})$ ). Then as the $\Lambda$ vary, we have

$$
\theta(L ; \tau)=\sum_{\Lambda} e\{\Lambda \tau\}
$$

When $n=1$ and $p$ is a prime not dividing the level $N$ of $L$, the Eichler Commutation Relation (see [8]; cf. [10]) says that if $\chi(p)=1$ then

$$
\theta(L ; \tau) \mid T(p)=\kappa \sum_{K} \theta\left(K^{1 / p} ; \tau\right)
$$

where $\kappa$ is an explicit constant, and $K$ varies over preimages in $L$ of all maximal totally isotropic subspaces of $L / p L$; here $K^{1 / p}$ refers to the lattice $K$ equipped with the scaled quadratic form $\frac{1}{p} Q$. ( $L / p L$ is a quadratic space with induced quadratic form $Q \bmod p$; a subspace $\bar{C}$ is totally isotropic if $Q(\bar{C})=0$ in $\mathbb{Z} / p \mathbb{Z}$.) If $\chi=1$, then $K^{1 / p} \in$ gen $L$, meaning that for all primes $q, \mathbb{Z}_{q} K^{1 / p} \simeq \mathbb{Z}_{q} L$, and consequently with

$$
\theta(\operatorname{gen} L)=\sum_{\operatorname{cls} L^{\prime} \in \operatorname{gen} L} \frac{1}{o\left(L^{\prime}\right)} \theta\left(L^{\prime}\right),
$$

$o\left(L^{\prime}\right)$ the order of the orthogonal group of $L^{\prime}$, we have

$$
\theta(\operatorname{gen} L) \mid T(p)=\left(p^{k-1}+1\right) \theta(\operatorname{gen} L) .
$$


If $\chi \neq 1$ then $\theta(\operatorname{gen} L)$ is an eigenform for some of the $T(p)$ and for all $T\left(p^{2}\right)$ ( $p$ a prime not dividing the level of $L)$. $(\theta(\operatorname{gen} L)$ is an eigenform for $T(p)$ if $\chi(p)=1$, and for all primes $q \mid N$, either $\left(\frac{p}{q}\right)=1$ or $\mathbb{Z}_{q} L$ has no modular components of odd rank.)

So now assume $2 \leq n \leq k$, and $p$ is a prime not dividing $N$, the level of $L$. We have $n+1$ Hecke operators associated to $p$, named $T(p), T_{j}\left(p^{2}\right)(1 \leq j \leq n)$. For $T$ one of these operators, there is an associated matrix $\delta$ so that

$$
F\left|T=p^{\eta} \sum_{\gamma} F\right| \delta^{-1} \gamma
$$

where $\gamma$ runs over $\left(\Gamma \cap \Gamma^{\prime}\right) \backslash \Gamma, \Gamma=\Gamma_{1}(N), \Gamma^{\prime}=\delta \Gamma \delta^{-1}$, and $p^{\eta}$ is a normalizing factor. Here $\delta=\left(\begin{array}{cc}p I_{n} & \\ & I_{n}\end{array}\right)$ and $\eta=n(k-n-1) / 2$ when $T=T(p)$;

$$
\delta=\left(\begin{array}{cccc}
p I_{j} & & & \\
& I_{n-j} & & \\
& & \frac{1}{p} I_{j} & \\
& & & I_{n-j}
\end{array}\right)
$$

and $\eta=0$ when $T=T_{j}\left(p^{2}\right)$. (Later we normalize particular linear combinations of the $T_{j}\left(p^{2}\right)$.)

Say $F$ is a degree $n$, weight $k$ Siegel modular form (with some level and character). For $C \in G L_{n}(\mathbb{Z}),\left(\begin{array}{ll}C^{-1} & \\ & { }^{t} C\end{array}\right) \in S p_{n}(\mathbb{Z})$; consequently we can write

$$
F(\tau)=\sum_{T} c(T) e\{T \tau\}=\sum_{\operatorname{cls} \Lambda} c(\Lambda) e^{*}\{\Lambda \tau\}
$$

where $\operatorname{cls} \Lambda$ varies over all isometry classes of even integral rank $n$ positive semidefinite lattices (oriented when $k$ is odd), $e^{*}\{\Lambda \tau\}=\sum_{C} e\left\{{ }^{t} C T C \tau\right\}$ where $\Lambda \simeq T$, $C \in O(T) \backslash G L_{n}(\mathbb{Z})$ if $k$ is even, and $C \in O^{+}(T) \backslash S L_{n}(\mathbb{Z})$ if $k$ is odd. (Here $O(T)$ is the orthogonal group of $T$.)

Theorem 1.1: Action of Hecke operators on Fourier coefficients.

(1) ([6]) The coefficient of $e^{*}\{\Lambda \tau\}$ in $F \mid T(p)$ is

$$
\sum_{p \Lambda \subseteq \Omega \subseteq \Lambda} p^{E(\Lambda, \Omega)} c\left(\Omega^{1 / p}\right)
$$

where $E(\Lambda, \Omega)=m(1) k+m(p)(m(p)+1) / 2-n(n+1) / 2, m(a)=m_{u l t}\{\Lambda: \Omega\}(a)$.

(2) ([5]) Set $\widetilde{T}_{j}\left(p^{2}\right)=p^{j(k-n-1)} \sum_{0 \leq \ell \leq j} \beta(n-\ell, j-\ell) T_{\ell}\left(p^{2}\right)$ where $\beta(m, t)=$ $\prod_{i=0}^{t-1} \frac{p^{m-i}-1}{p^{t-i}-1}$, the number of $t$-dimensional subspaces of an $m$-dimensional space over $\mathbb{Z} / p \mathbb{Z}$, and $T_{0}\left(p^{2}\right)=1$. The coefficient on $e^{*}\{\Lambda \tau\}$ in $F \mid \widetilde{T}_{j}\left(p^{2}\right)$ is

$$
\sum_{p \Lambda \subseteq \Omega \subseteq \frac{1}{p} \Lambda} p^{E_{j}(\Lambda, \Omega)} \alpha_{j}(\Lambda, \Omega) c(\Omega) .
$$


Here $\alpha_{j}(\Lambda, \Omega)$ is the number of codimension $n-j$ totally isotropic subspaces of $\Lambda \cap \Omega / p(\Lambda+\Omega)$, and $E_{j}(\Lambda, \Omega)=k(m(1 / p)-m(p)+j)+m(p)(m(p)+m(1)+1)+$ $m_{j}(1)\left(m_{j}(1)+1\right) / 2-j(n+1), m_{j}(1)=m(1)-n+j$.

In [5] we proved this by computing a set of coset representatives giving the action of each Hecke operator. We encountered incomplete character sums, so we introduced the modified operators $\widetilde{T}_{j}\left(p^{2}\right)$. In this paper, we apply these coset representatives to $\theta(L)$, obtaining

Theorem 2.1. Let $p$ be a prime with $\chi(L)=1$. Set

$$
\beta(m, r)=\prod_{i=0}^{r-1} \frac{\left(p^{m-i}-1\right)}{\left(p^{r-i}-1\right)}
$$

(the number of $r$-dimensional subspaces of an $m$-dimensional space over $\mathbb{Z} / p \mathbb{Z}$ ), and set

$$
\epsilon(m, r)=\prod_{i=0}^{r-1}\left(p^{m+i}+1\right) .
$$

(1) $\theta(L ; \tau) \mid T(p)=\frac{1}{\epsilon(0, k-n)} \sum_{K} \theta(K ; \tau / p)$ where $K$ varies over the preimages in $L$ of all maximal, totally isotropic subspaces of $L / p L$.

(2) Fix $j$ and set

$$
\begin{aligned}
& u_{i}=u_{i}(j)=(-1)^{i} p^{i(i-1) / 2} \beta(n-j+i, i), \\
& v_{i}=v_{i}(j)=(-1)^{i} \beta(k-n+i-1, i) \epsilon(k-j, i) .
\end{aligned}
$$

Then with

$$
T_{j}^{\prime}\left(p^{2}\right)=\sum_{i=0}^{j} u_{i} \widetilde{T}_{j}\left(p^{2}\right)
$$

we have

$$
\theta(L) \mid T_{j}^{\prime}\left(p^{2}\right)=\sum_{i=0}^{j} v_{i}\left(\sum_{K_{j-i}} \theta\left(K_{j-i}\right)\right)
$$

where $K_{j-i}$ varies over all lattices $K$ satisfying $p L \subseteq K \subseteq \frac{1}{p} L$ mult $_{\{L: K\}}\left(\frac{1}{p}\right)=$ $\operatorname{mult}_{\{L: K\}}(p)=j-i, K \in$ genL.

Note that the sets $\left\{T(p), T_{j}\left(p^{2}\right)\right\},\left\{T(p), \widetilde{T}_{j}\left(p^{2}\right)\right\},\left\{T(p), T_{j}^{\prime}\left(p^{2}\right)\right\}(0 \leq j \leq n)$ generate the same (local) Hecke algebra.

Averaging over the isometry classes in the genus of $L$, we get the average theta series

$$
\theta(\operatorname{gen} L)=\frac{1}{\operatorname{mass} L} \sum_{\operatorname{cls} L^{\prime}} \frac{1}{o\left(L^{\prime}\right)} \theta\left(L^{\prime}\right)
$$

where $\operatorname{cls} L^{\prime}$ varies over gen $L, o\left(L^{\prime}\right)$ is the order of the isometry group of $L^{\prime}$, and

$$
\operatorname{mass} L=\sum_{\operatorname{cls} L^{\prime}} \frac{1}{o\left(L^{\prime}\right)} .
$$

Also, averaging across the equation in the above theorem, we get: 
Corollary 2.2. Let $p$ be a prime such that $\chi(p)=1$. Then

$$
\theta(g e n L ; \tau) \mid T(p)=\epsilon(k-n, n) \theta(g e n L ; \tau) .
$$

Also,

$$
\theta(\text { genL }) \mid T_{j-i}^{\prime}\left(p^{2}\right)=\lambda_{j}\left(p^{2}\right) \theta(\text { genL })
$$

where

$$
\lambda_{j}\left(p^{2}\right)=p^{j(k-n)+j(j-1) / 2} \beta(n, j) \epsilon(k-j, j) .
$$

To prove the theorem, we apply to $\theta(L)$ the coset representatives giving the action of $T(p)$ and of $\widetilde{T}_{j}\left(p^{2}\right)$. Initially, we have

$$
\begin{aligned}
\theta(L ; \tau) \mid T(p) & =\sum_{\Lambda \subseteq L}\left(\sum_{p \Lambda \subseteq \Omega \subseteq \Lambda} p^{E(\Omega, \Lambda)}\right) e(\Omega \tau / p) \\
& =\sum_{\substack{\Omega \subseteq L \\
\Omega^{1 / p} \text { integral }}}\left(\sum_{p \Omega \subseteq p \Lambda \subseteq \Omega} p^{E(\Omega, \Lambda)}\right) e\{\Omega \tau / p\},
\end{aligned}
$$

and

$$
\begin{aligned}
\theta(L ; \tau) \mid \widetilde{T}_{j}\left(p^{2}\right) & =\sum_{\Lambda \subseteq L}\left(\sum_{\substack{p \Lambda \subseteq \Omega \subseteq \frac{1}{p} \Lambda \\
\Omega \text { integral }}} p^{E_{j}(\Omega, \Lambda)} \alpha_{j}(\Lambda, \Omega)\right) e\{\Omega \tau\} \\
& =\sum_{\substack{\Omega \subseteq \frac{1}{p} L \\
\Omega \text { integral }}}\left(\sum_{p \Omega \subseteq \Lambda \subseteq\left(\frac{1}{p} \Omega \cap L\right)} p^{E_{j}(\Omega, \Lambda)} \alpha_{j}(\Lambda, \Omega)\right) e\{\Omega \tau\}
\end{aligned}
$$

Fixing some integral $\Omega \subseteq L$ or $\Omega \subseteq \frac{1}{p} L$, we will prove this by using elementary lattice techniques to construct all the $\Lambda$ in the inner sums, as we simultaneously compute $\alpha_{j}(\Lambda, \Omega)$. First, we decompose $\Omega$ as $\frac{1}{p} \Omega_{0} \oplus \Omega_{1} \oplus p \Omega_{2}, \Omega_{i} \subseteq L$, with $\Omega_{0}, \Omega_{1}$ primitive in $L$ mod $p$, meaning $\left(\Omega_{0} \oplus \Omega_{1}\right) \cap p L=p\left(\Omega_{0} \oplus \Omega_{1}\right)$. Set $\varphi_{\ell}(\bar{V})=$ the number of $\ell$-dimensional totally isotropic subspaces of $\bar{V}$, where $\bar{V}$ is a quadratic space over $\mathbb{Z} / p \mathbb{Z}$. (We agree that $\epsilon(m, 0)=1$.) Also set

$$
A_{j}(\ell, t, \Omega)=p^{E} \varphi_{\ell}\left(\bar{\Omega}_{1}\right) \epsilon\left(k-j+t, j-r_{0}-\ell-t\right) \beta\left(r_{2}, j-r_{0}-\ell-t\right)
$$

where $\bar{\Omega}_{1}=\Omega_{1} / p \Omega_{1}$ and

$$
\begin{gathered}
E=E_{j}(\ell, t, \Omega)=(k-n)\left(j-r_{0}-t\right)+\left(j-r_{0}-t\right)\left(j-r_{0}-t-1\right) / 2 \\
+\ell\left(\ell+n-j-r_{1}+t\right) .
\end{gathered}
$$

Then, as we prove in $\S 3$, we get: 
Proposition 3.1. (1) Write

$$
\theta(L ; \tau) \mid T(p)=\sum_{\Omega} c^{*}(\Omega) e\{\Omega \tau / p\}
$$

where $\Omega \subseteq L$ varies over all lattices with (formal) rank $n$ and $Q(\Omega) \equiv 0(\bmod p)$; write $\Omega=\Omega_{1} \oplus p \Omega_{2}, \Omega_{i} \subseteq L, \Omega_{1}$ primitive in $L$ modulo $p$, meaning $\Omega_{1} \cap p L=p \Omega_{1}$. Then

$$
c^{*}(\Omega)=\epsilon\left(k-n, r_{2}\right)
$$

where $r_{2}=\operatorname{rank} \Omega_{2}$.

(2) Write

$$
\theta(L) \mid \widetilde{T}_{j}\left(p^{2}\right)=\sum_{\Omega} c_{j}^{*}(\Omega) e\{\Omega \tau\}
$$

where $\Omega$ varies over all integral lattices $\Omega \subseteq \frac{1}{p} L$ with (formal) rank $n$. Decompose $\Omega$ as $\frac{1}{p} \Omega_{0} \oplus \Omega_{1} \oplus p \Omega_{1}$ where $\Omega_{i} \subseteq L, \Omega_{0}, \Omega_{1}$ primitive in $L$ modulo $p$, meaning $\left(\Omega_{0} \oplus \Omega_{1}\right) \cap p L=p\left(\Omega_{0} \oplus \Omega_{1}\right)$. Then

$$
c_{j}^{*}(\Omega)=\sum_{\ell, t} A_{j}(\ell, t, \Omega) \beta(n-j+t, t)
$$

where $0 \leq \ell \leq j-r_{0}$ and $0 \leq t \leq j-r_{0}-\ell, r_{0}=\operatorname{rank} \Omega_{0}$.

Proposition 3.2. Suppose $1 \leq j \leq n \leq k$ and $\chi(p)=1$ (so $L / p L$ is hyperbolic).

(1) Let $K$ vary over the preimages in $L$ of all maximal, totally isotropic subspaces of $L / p L$. Then

$$
\sum_{K} \theta(K ; \tau / p)=\epsilon(0, k-n) \sum_{\Omega} \epsilon\left(k-n, r_{2}\right) e\{\Omega \tau / p\}
$$

where $\Omega$ vary as in Proposition 3.1 (1), $r_{2}=\operatorname{rank} \Omega_{2}$.

(2) Let $K_{j}$ vary over all lattices such that $p L \subseteq K_{j} \subseteq \frac{1}{p} L, \operatorname{mult}_{\left\{L: K_{j}\right\}}\left(\frac{1}{p}\right)=$ mult $_{\left\{L: K_{j}\right\}}(p)=j$, and $K_{j} \in$ genL. Write

$$
\sum_{K_{j}} \theta\left(K_{j}\right)=\sum_{\Omega} b_{j}(\Omega) e\{\Omega \tau\}
$$

where $\Omega$ varies as in Proposition 3.1 (2). Then

$$
b_{j}(\Omega)=\sum_{\ell, t} A_{j}(\ell, t, \Omega) p^{t(t-1) / 2} \beta(k-n, t) \epsilon(k-j, t)
$$

where $0 \leq \ell \leq j-r_{0}$ and $0 \leq t \leq j-r_{0}-\ell$. The number of $K_{j}$ in this sum is $p^{j(j-1) / 2} \beta(k, j) \epsilon(k-j, j)$.

Critical to proving Proposition 3.2 will be the following: 
Lemma 4.1 (Reduction Lemma). Let $U$ be a dimension $d$ space over $\mathbb{Z} / p \mathbb{Z}, \mathbb{H}$ a hyperbolic plane (so $\mathbb{H} \simeq\left(\begin{array}{ll}0 & 1 \\ 1 & 0\end{array}\right)$ ). Then:

$$
\varphi_{\ell}\left(U \perp \mathbb{H}^{t}\right)=\sum_{0 \leq r \leq t} p^{(t-r)(\ell-r)} \epsilon(d+t-\ell, r) \beta(t, r) \varphi_{\ell-r}(U) .
$$

(Note that the summand on $r$ is 0 unless $r \leq t, \ell$, and thus we can let $r$ vary subject to $r \geq 0$.)

Remark. In [4], Freitag used the theory of singular forms to give a simple description of the action of $T(p)$ on theta series of level 1. Then in [11] and [12] (cf. [9]), Yoshida used representation theory to generalize and extend Freitag's result (omitting the spherical harmonics Freitag allowed) to describe the action of $T(p)$ and of $T_{j}\left(p^{2}\right)$ on theta series of arbitrary level $(p \nmid N)$. Yoshida's formula is presented in a simple form when $k=n$. While our formulas are presented more simply when $k \neq n$ and our methods are more elementary than those of Yoshida, our methods only allow us to treat $n \leq k$ whereas Yoshida apparently can treat $n \leq 2 k$. Also, in this paper we treat only the case $\chi(p)=1$; in current work we are using the methods of this paper to treat the case $\chi(p)=-1$. We are also investigating the case $k<n \leq 2 k$, seeking elementary arguments that yield explicit eigenvalues.

The reader is referred to [1] and [3] for facts about Siegel modular forms, and to [2] and [7] for facts about quadratic forms.

\section{Proofs of Theorem 2.1 And Corollary 2.2}

Proof of Theorem 2.1. (1) This is easily seen from Propositions 3.1 and 3.2.

(2) The theorem states that for all $\Omega$,

$$
\begin{aligned}
& \sum_{i=0}^{j}(-1)^{i} p^{i(i+1) / 2} \beta(n-j+i, i) c_{j-i}^{*}(\Omega) \\
& \quad=\sum_{i=0}^{j}(-1)^{i} \beta(k-n+i-1, i) \epsilon(k-j, i) b_{j-i}(\Omega) .
\end{aligned}
$$

To establish this, we set $S_{0}(\Omega)=c_{j}^{*}(\Omega)-b_{j}(\Omega)$, and for $q \geq 1$,

$$
\begin{gathered}
S_{q}(\Omega)=S_{q-1}(\Omega)+(-1)^{q} p^{q(q+1) / 2} \beta(n-j+q, q) c_{j-q}^{*}(\Omega) \\
+\beta(k-n+q-1, q) \epsilon(k-j, q) b_{j-q}(\Omega) .
\end{gathered}
$$

We want to show $S_{j}=0$. We use induction on $q$ to show that for $q<j$,

$$
\begin{aligned}
& S_{q}(\Omega)=\sum_{\ell, t} A_{j-(q+1)}(\ell, t, \Omega) \beta(t+q, q) \\
& \cdot\left[(-1)^{q} p^{q(q+1) / 2} \beta(n-j+t+q+1, t+q+1)\right. \\
&\left.\quad-(-1)^{q} p^{t(t+1) / 2} \beta(k-n+q, t+q+1) \epsilon(k-j, t+q+1)\right]
\end{aligned}
$$


where $0 \leq \ell \leq j-(q+1)-r_{0}$ and $0 \leq t \leq j-(q+1)-r_{0}-\ell$. Using the formulas for $c_{j}^{*}(\Omega)$ and $b_{j}(\Omega)$, we get

$$
S_{0}(\Omega)=\sum_{\ell, t} A_{j}(\ell, t, \Omega)\left[\beta(n-j+t, t)-p^{t(t-1) / 2} \beta(k-n, t) \epsilon(k-j, t)\right] .
$$

When $t=0$, the summand is 0 , and necessarily $\mathrm{t}=0$ when $\ell=j-r_{0}$. Replacing $t$ by $t+1$ and using that $A_{j}(\ell, t+1, \Omega)=A_{j-1}(\ell, t, \Omega)$ we establish the claim for $q=0$.

Now assume the formula holds for $S_{q}(\Omega)$; we evaluate $S_{q+1}(\Omega)$ using the above assumption and our formulas for $c_{m}^{*}(\Omega), b_{m}(\Omega)$. We can write $S_{q+1}(\Omega)$ as a sum on $\ell$ and $t$; the summand is 0 when $t=0$, and we necessarily have $t=0$ when $\ell=j-r_{0}$. Thus we can write $S_{q+1}(\Omega)$ as a sum on $\ell<j-r_{0}, t>0$. By Lemma $5.1(\mathrm{~b})$

$$
\begin{aligned}
& \beta(n-j+q+1, q+1) \beta(n-j+t+q+1, t) \\
& \quad=\beta(n-j+t+q+1, t+q+1) \beta(t+q+1, t) .
\end{aligned}
$$

Also, one can easily verify that

$$
\beta(t+q+1, t)-\beta(t+q, q)=p^{q+1} \beta(t+q, q+1) .
$$

We have $\epsilon(j-j, q+1) \epsilon(k-j+q+1, t)=\epsilon(k-j, t+q+1)$, and by Lemma 5.1(d),

$$
\begin{aligned}
& p^{t} \beta(k-n+q, t+q+1, t+q+1) \beta(t+q, q)-\beta(k-n+q, q+1) \beta(k-n, t) \\
& \quad=-\beta(k-n+q+1, t+q+1) \beta(t+q, q+1) .
\end{aligned}
$$

Using these identities, replacing $t$ by $t+1$, and using that $A_{j-(q+1)}(\ell, t+1, \Omega)=$ $A_{j-(q+2)}(\ell, t, \Omega)$ yields the claim for $q<j$. In particular,

$$
S_{j-1}(\Omega)=(-1)^{j-1} p^{j(j-1) / 2} \beta(n, j)-(-1)^{j-1} \beta(k-n+j-1, j) \epsilon(k-j, j) .
$$

We have $\theta(L) \mid \widetilde{T}_{0}\left(p^{2}\right)=\theta(L)$ and $K_{0}=L$, so $c_{0}^{*}(\Omega)=b_{0}(\Omega)=1$, and thus the inductive definition shows $S_{j}(\Omega)=0$. This proves the theorem.

Proof of Corollary 2.2. (1) This is virtually identical, although a little simpler, than the proof of (2), and so we omit this proof.

(2) With $o\left(L^{\prime}\right)$ the order of the orthogonal group for $L^{\prime}$, we set

$$
\theta(\operatorname{gen} L)=\frac{1}{\operatorname{mass} L} \sum_{L^{\prime}} \frac{1}{o\left(L^{\prime}\right)} \theta\left(L^{\prime}\right)
$$

where $\operatorname{cls} L^{\prime}$ varies over the isometry classes in gen $L$, and mass $L=\sum_{L^{\prime}} \frac{1}{o\left(L^{\prime}\right)}$. Consider the effect averaging over gen $L$ has on $\sum_{K_{m}} \theta\left(K_{m}\right)$ where $K_{m}$ varies over all lattices in gen $L$ with $p L \subseteq K_{m} \subseteq \frac{1}{p} L$ and $\operatorname{mult}_{\left\{L: K_{m}\right\}}(1 / p)=\operatorname{mult}_{\left\{L: K_{m}\right\}}(p)=$ 
$m$. First, note that the condition $\operatorname{mult}_{\left\{L: K_{m}\right\}}(1 / p)=m$ is automatically met when the other conditions are met. Then, note that we can classify the $K_{m}$ into isometry classes; so with $K_{m} \in \operatorname{cls} K$, we have an isometry $\sigma$ so that $\sigma(K)=K_{m}$, meaning $\operatorname{mult}_{\{L: \sigma K\}}(1 / p)=\operatorname{mult}_{\{L: \sigma K\}}(p)=m$. Given isometries $\sigma, \sigma^{\prime}, \sigma K=\sigma^{\prime} K$ if and only if $\sigma^{-1} \sigma^{\prime} \in O(K)$, the orthogonal group of $K$. Thus the number of $\sigma$ that map $K$ to the same lattice is $o(K)$, and so with $L$ fixed,

$$
\sum_{K_{m}} \theta\left(K_{m}\right)=\sum_{\mathrm{cls} K} \frac{\#\left\{\sigma: p L \subseteq K_{m} \subseteq \frac{1}{p} L, \operatorname{mult}_{\{L: \sigma K\}}(p)=m\right\}}{o(K)} \theta(K) .
$$

Now take the identity of the preceeding theorem and average both sides over $\operatorname{cls} L^{\prime} \in \operatorname{gen} L$. So with $K_{m}^{\prime}$ varying over all lattices in gen $L^{\prime}=\operatorname{gen} L$ with

$$
\operatorname{mult}_{\left\{L^{\prime}: K_{m}^{\prime}\right\}}(1 / p)=\operatorname{mult}_{\left\{L^{\prime}: K_{m}^{\prime}\right\}}(p)=m,
$$

we have

$$
\begin{aligned}
\sum_{\operatorname{cls} L^{\prime}} & \frac{1}{o\left(L^{\prime}\right)} \sum_{K_{m}^{\prime}} \theta\left(K_{m}^{\prime}\right) \\
& =\sum_{\operatorname{cls} L^{\prime}, \operatorname{cls} K^{\prime}} \frac{\#\left\{\sigma: p L^{\prime} \subseteq \sigma K^{\prime} \subseteq \frac{1}{p} L^{\prime}, \operatorname{mult}_{\left\{L^{\prime}: \sigma K^{\prime}\right\}}(p)=m\right\}}{o\left(L^{\prime}\right) o\left(K^{\prime}\right)} \theta\left(K^{\prime}\right) \\
& =\sum_{\operatorname{cls} K^{\prime}}\left(\sum_{\operatorname{cls} L^{\prime}} \frac{\#\left\{\sigma: p K^{\prime} \subseteq \sigma L^{\prime} \subseteq \frac{1}{p} K^{\prime}, \operatorname{mult}_{\left\{K^{\prime}: \sigma L^{\prime}\right\}}(p)=m\right\}}{o\left(L^{\prime}\right)}\right) \frac{1}{o\left(K^{\prime}\right)} \theta\left(K^{\prime}\right)
\end{aligned}
$$

(the last equality follows from replacing $\sigma$ by $\sigma^{-1}$ ). The inner sum on $L^{\prime}$ is the number of lattices $K_{m} \in \operatorname{gen} L$ so that $p K^{\prime} \subseteq K_{m} \subseteq \frac{1}{p} K^{\prime}$ and $\operatorname{mult}_{\left\{K^{\prime}: K_{m}\right\}}(p)=m$, and by Proposition 3.2, this is $p^{m(m-1) / 2} \varphi_{m}(L / p L)$, and since $L / p L \simeq \mathbb{H}^{k}$, this is

$$
p^{m(m-1) / 2} \beta(k, m) \epsilon(k-m, m) .
$$

Thus

$$
\lambda_{j}=\epsilon(k-j, j) \sum_{i=0}^{j}(-1)^{i} p^{(j-i)(j-i-1) / 2} \beta(k, j-i) \beta(k-n+i-1, i) .
$$

We claim that the sum on $i$ is equal to

$$
p^{j(k-n)+j(j-1) / 2} \beta(n, j) ;
$$

we argue by induction on $k(k \geq n \geq j \geq 0)$. For $k=0$, this is trivial. So assume $k>0$ and the formula holds for $k-1$ whenever $k-1 \geq n \geq j \geq 0$. When $j=k$ 
or $j=0$ the claim is trivially verified. Thus we now consider $k>j>0$. We make use of the easily verified identity

$$
\beta(m, r)=\beta(m-1, r)+p^{m-r} \beta(m-1, r-1)
$$

(where $m \geq r \geq 1$ ). This allows us to rewrite:

$$
\begin{aligned}
& \sum_{i=0}^{j}(-1)^{i} p^{(j-i)(j-i-1) / 2} \beta(k, j-i) \beta(k-n+i-1, i) \\
& \quad=\sum_{i=0}^{j}(-1)^{i} p^{(j-i)(j-i-1) / 2} \beta(k-1, j-i) \beta(k-n+i-1, i) \\
& \quad+p^{k-1} \sum_{i=0}^{j-1}(-1)^{i} p^{(j-i-1)(j-i-2) / 2} \beta(k-1, j-i-1) \beta(k-n+i-1, i)
\end{aligned}
$$

and then, by the induction hypothesis

$$
\begin{aligned}
& =p^{j(k-n)+j(j-1) / 2} \beta(n-1, j)+p^{k-1+(j-1)(k-n)+(j-1)(j-2) / 2} \beta(n-1, j-1) \\
& =p^{j(k-n)+j(j-1) / 2}\left[\beta(n-1, j)+p^{n-j} \beta(n-1, j-1)\right]
\end{aligned}
$$

and again using our identity (with $n$ replaced by $n-1$ )

$$
=p^{j(k-n)+j(j-1) / 2} \beta(n, j) .
$$

\section{Proofs of Propositions 3.1 And 3.2}

Proof of Proposition 3.1. (1) From [5] we know

$$
\begin{aligned}
& \theta(L ; \tau) \mid T(p) \\
& \quad=p^{n(k-n-1) / 2} \sum_{B, C} \theta(L ; \tau)\left(\begin{array}{lll}
\frac{1}{p} I_{n} & \\
& I_{n}
\end{array}\right)\left(\begin{array}{cccc}
I_{d} & & B & \\
& p I_{n-d} & & 0 \\
& & I_{d} & \\
& & \\
& I_{n-d}
\end{array}\right)\left(\begin{array}{ll}
C^{-1} & \\
& { }^{t} C
\end{array}\right) \\
& \quad p^{-n(n+1) / 2} p^{k(n-d)} e\left\{T\left[G\left(\begin{array}{ll}
I_{d} & \\
& p I_{n-d}
\end{array}\right) C^{-1}\right] \tau / p\right\} .
\end{aligned}
$$

Here $C=C\left(\Delta^{\prime}\right)$ varies over all $\Delta^{\prime}, p \Delta \subseteq \Delta^{\prime} \subseteq \Delta, \Delta$ a fixed reference lattice of rank $n, \Delta^{\prime}=\Delta C\left(\begin{array}{cc}p I_{d} & \\ & I_{n-d}\end{array}\right)$; for each such $C, B$ varies over all integral, symmetric, $d \times d$ matrices modulo $p ; \Lambda$ varies over all sublattices of $L$ with (formal) rank $n$, $\Lambda \simeq T$; and $G$ varies over $G L_{n}(\mathbb{Z})$ (or, if $k$ is odd, $\Lambda$ is equipped with an orientation and $G$ varies over $S L_{n}(\mathbb{Z})$ ). The sum on $B$ is either 0 or $p^{d(d+1) / 2}$, depending on whether the upper left $d \times d$ block of $T[G]$ is 0 modulo $p$. 
Fix $d$ and let $\mathcal{G}$ be the subgroup of $G L_{n}(\mathbb{Z})$ (or, if $k$ is odd, of $S L_{n}(\mathbb{Z})$ ) of all matrices of the form $\left(\begin{array}{cc}E_{1} & E_{2} \\ p E_{3} & E_{4}\end{array}\right)$ with $E_{3} \in \mathbb{Z}^{n-d, d}$. Then the cosets of $G L_{n}(\mathbb{Z}) / \mathcal{G}$ (or of $S L_{n}(\mathbb{Z}) / \mathcal{G}$ ) correspond to the lattices $\Omega$ where $p \Lambda \subseteq \Omega \subseteq \Lambda,[\Lambda: \Omega]=p^{n-d}$, as the matrices $G$ so that

$$
\Omega=\Lambda G\left(\begin{array}{ll}
I_{d} & \\
& p I_{n-d}
\end{array}\right)
$$

comprise one coset. Similarly, the matrices $C=C\left(\Delta^{\prime}\right)$ give us a complete list of coset representatives for $G L_{n}(\mathbb{Z}) /{ }^{t} \mathcal{G}$ (or for $S L_{n}(\mathbb{Z}) /{ }^{t} \mathcal{G}$ ), as $\Delta^{\prime}$ varies subject to $p \Delta \subseteq \Delta^{\prime} \subseteq \Delta,\left[\Delta: \Delta^{\prime}\right]=p^{d}$ (so $\Delta^{\prime}=\Delta C\left(\begin{array}{cc}p I_{d} & \\ & I_{n-d}\end{array}\right)$ for some $C$ ). Consequently as $C$ varies and as $G^{\prime}$ varies over $\mathcal{G}, C^{t} G^{\prime}$ varies over $G L_{n}(\mathbb{Z})\left(\right.$ or $S L_{n}(\mathbb{Z})$ ), and thus so does ${ }^{t} G^{\prime} C^{-1}$. Hence as $G$ varies over $G L_{n}(\mathbb{Z})$ (or $S L_{n}(\mathbb{Z})$ ),

$$
\begin{gathered}
\sum_{G, C} e\left\{T\left[G\left(\begin{array}{ll}
I_{d} & \\
& p I_{n-d}
\end{array}\right) C^{-1}\right] \tau / p\right\} \\
=\sum_{\Omega} \sum_{G^{\prime}, C} e\left\{T^{\prime}\left[{ }^{t} G^{\prime} C^{-1}\right] \tau / p\right\}
\end{gathered}
$$

where $G^{\prime}$ varies over $\mathcal{G}, \Omega$ varies over all lattices subject to $p \Lambda \subseteq \Omega \Lambda,[\Lambda: \Omega]=p^{n-d}$, and $\Omega \simeq T^{\prime}$. Thus

$$
\theta(L ; \tau) \mid T(p)=p^{-n(n+1) / 2} \sum_{\Lambda, \Omega} p^{k(n-d)+d(d+1) / 2} e\{\Omega \tau / p\}
$$

where $\Lambda$ varies over all sublattices of $L$ with (formal) rank $n, \Omega$ is as above with $Q(\Omega) \equiv 0(\bmod p)$. So $c^{*}(\Omega)=p^{-n(n+1) / 2} \sum_{\Lambda} p^{k(n-d)+d(d+1) / 2}$ where $\Lambda$ varies over all lattices with (formal) rank $n, \Omega \subseteq \Lambda \subseteq\left(\frac{1}{p} \Omega \cap L\right)$. Write $\Omega=\Omega_{1} \oplus p \Omega_{2}$ where $\Omega_{1}, \Omega_{2} \subseteq L, \Omega_{1} \cap p L=p \Omega_{1}$. Thus $\frac{1}{p} \Omega \cap L=\Omega_{1} \oplus \Omega_{2}$, so the $\Lambda$ correspond to rank $n-d$ subspaces of $\Omega_{2} / p \Omega_{2}$ where $[\Lambda: \Omega]=p^{n-d}$. Thus with $r_{2}=\operatorname{rank} \Omega_{2}$, and recalling that $\beta\left(r_{2}, r_{2}-n+d\right)=\beta\left(r_{2}, n-d\right)$,

$$
c^{*}(\Omega)=p^{-n(n+1) / 2} \sum_{d=0}^{r_{2}} p^{k(n-d)+d(d+1) / 2} \beta\left(r_{2}, n-d\right)
$$

(and replacing $n-d$ by $d$ )

$$
=\sum_{d=0}^{r_{2}} p^{(k-n) d+d(d-1) / 2} \beta\left(r_{2}, d\right)
$$

(and then by Lemma 5.2)

$$
=\epsilon\left(k-n, r_{2}\right) .
$$


(2) We have

$$
\theta(L ; \tau) \mid \widetilde{T}_{j}\left(p^{2}\right)=\sum_{\substack{\Omega \subseteq \frac{1}{p} L \\ \Omega \text { integral }}}\left(\sum_{p \Omega \subseteq \Lambda \subseteq\left(\frac{1}{p} \Omega \cap L\right)} p^{E_{j}(\Omega, \Lambda)} \alpha_{j}(\Lambda, \Omega)\right) e\{\Omega \tau\} .
$$

Fix some integral $\Omega \subseteq \frac{1}{p} L$; decompose $\Omega$ as $\frac{1}{p} \Omega_{0} \oplus \Omega_{1} \oplus p \Omega_{2}, \Omega_{i} \subseteq L$, with $\Omega_{0}, \Omega_{1}$ primitive in $L$ mod $p$, meaning $\left(\Omega_{0} \oplus \Omega_{1}\right) \cap p L=p\left(\Omega_{0} \oplus \Omega_{1}\right)$.

We proceed by constructing all $\Lambda$ in the above sum on $\Lambda$, sorted according to their invariant factors $\{\Omega: \Lambda\}$ in $\Omega$, simultaneously constructing all the subspaces of $\Lambda \cap \Omega / p(\Lambda+\Omega)$ counted by $\alpha_{j}(\Omega, \Lambda)$.

We have $\Omega \subseteq \frac{1}{p} L$ with $\Omega$ integral, so we can decompose $\Omega$ as $\Omega=\frac{1}{p} \Omega_{0} \oplus \Omega_{1} \oplus p \Omega_{2}$ where $\Omega_{0}, \Omega_{1}, \Omega_{2} \subseteq L$ with $\Omega_{0}, \Omega_{1}$ primitive in $L$ modulo $p$, meaning $\left(\Omega_{0} \oplus \Omega_{1}\right) \cap$ $p L=p\left(\Omega_{0} \oplus \Omega_{1}\right)$. Since $\Omega$ is integral, we must have $Q\left(\Omega_{0}\right) \simeq 0\left(\bmod p^{2}\right)$ and $B\left(\Omega_{0}, \Omega_{1}\right) \simeq 0(\bmod p)$. Note that $\Omega_{0}$ is only well-determined up to $p\left(\Omega_{1} \oplus \Omega_{2}\right)$ and $\Omega_{0} \oplus \Omega_{1}$ is only well-determined up to $p \Omega_{2}$.

Define $\Delta=\frac{1}{p} \Omega \cap L$; so $\Delta=\Omega_{0} \oplus \Omega_{1} \oplus \Omega_{2}$. Also, $\Omega \cap L=\Omega \cap \Delta=\Omega_{0} \oplus \Omega_{1} \oplus p \Omega_{2}$, and $p(\Omega+\Delta)=\Omega_{0} \oplus p \Omega_{1} \oplus p \Omega_{2}$. Given $\Lambda$ with $p \Omega \subseteq \Lambda \subseteq\left(\frac{1}{p} \Omega \cap L\right)$, we must have

$$
\Lambda=\Omega_{0} \oplus\left(\Lambda_{1} \oplus p \Lambda_{1}^{\prime}\right) \oplus\left(\Lambda_{2} \oplus p \Lambda_{2}^{\prime} \oplus p^{2} \Lambda_{2}^{\prime \prime}\right)
$$

where $\Lambda_{1} \oplus \Lambda_{1}^{\prime}=\Omega_{1}, \Lambda_{2} \oplus \Lambda_{2}^{\prime} \oplus \Lambda_{2}^{\prime \prime}=\Omega_{2}$. Notice such $\Lambda$ is necessarily integral.

We now proceed to construct all such $\Lambda$, simultaneously counting the totally isotropic, co-dimension $n-j$ subspaces of

$$
\Lambda \cap \Omega / p(\Lambda+\Omega) \simeq\left(\Lambda_{1} \oplus p \Lambda_{2}^{\prime}\right) / p\left(\Lambda_{1} \oplus p \Lambda_{2}^{\prime}\right)
$$

Let $r_{i}=\operatorname{rank} \Omega_{i}, d_{1}+d_{1}^{\prime}=r_{1}, d_{2}+d_{2}^{\prime}+d_{2}^{\prime \prime}=r_{2}$. We will construct all $\Lambda$ so that when decomposed as above, $d_{i}=\operatorname{rank} \Lambda_{i}, d_{i}^{\prime}=\operatorname{rank} \Lambda_{i}^{\prime}$, and $d_{2}^{\prime \prime}=\operatorname{rank} \Lambda_{2}^{\prime \prime}$.

In $\Delta / p \Delta$, extend $\overline{\Omega \cap \Delta}=\overline{\Omega_{0} \oplus \Omega_{1}}$ to $\overline{\Omega_{0} \oplus \Omega_{1} \oplus \Delta_{2}}$ with $\operatorname{dim} \Delta_{2}=d_{2}+d_{2}^{\prime}$. (We have $\beta\left(r_{2}, d_{2}+d_{2}^{\prime}\right)=\beta\left(r_{2}, d_{2}^{\prime \prime}\right)$ choices.) Let

$$
\begin{aligned}
\Delta^{\prime} & =\text { preimage in } \Delta \text { of } \overline{\Omega_{0} \oplus \Omega_{1} \oplus \Delta_{2}} \\
& =\Omega_{0} \oplus \Omega_{1} \oplus\left(\Delta_{2} \oplus p \Lambda_{2}^{\prime \prime}\right)
\end{aligned}
$$

where $\operatorname{dim} p \Lambda_{2}^{\prime \prime}=d_{2}^{\prime \prime}$. In $\Delta^{\prime} / p \Delta^{\prime}$, extend $\bar{\Omega}_{0}=\overline{p \Omega}$ to

$$
\overline{\Omega_{0} \oplus U} \subseteq \overline{\Omega \cap \Delta}=\overline{\Omega_{0} \oplus \Omega_{1} \oplus p \Lambda_{2}^{\prime \prime}}
$$

so that $\bar{U}$ is totally isotropic of dimension $\ell$ and independent of $\overline{p \Delta}=\overline{p \Lambda_{2}^{\prime \prime}}$ (later we let $\ell$ vary). (We have $\varphi_{\ell}\left(\bar{\Omega}_{1}\right) p^{\ell d_{2}^{\prime \prime}}$ choices.) Now extend $\overline{\Omega_{0} \oplus U}$ to $\overline{\Omega_{0} \oplus \Lambda_{1}} \subseteq \overline{\Omega \cap \Delta}$ so that $\operatorname{dim} \bar{\Lambda}_{1}=d_{1}$ with $\bar{\Lambda}_{1}$ independent of $\overline{p \Delta}$. (We have $\left.\beta\left(r_{1}-\ell, d_{1}-\ell\right) p^{(} d_{1}-\ell\right) d_{2}^{\prime \prime}$ 
choices.) Extend $\overline{\Omega_{0} \oplus \Lambda_{1}}$ to $\overline{\Omega_{0} \oplus \Lambda_{1} \oplus \Lambda_{2}}$ with $\operatorname{dim} \bar{\Lambda}_{2}=d_{2}$ and $\bar{\Lambda}_{2}$ independent of $\overline{\Omega \cap \Delta}=\overline{\Omega_{0} \oplus \Omega_{1} \oplus p \Lambda_{2}^{\prime \prime}}$. (We have $\beta\left(d_{2}+d_{2}^{\prime}, d_{2}\right) p^{d_{2}\left(d_{1}^{\prime}+d_{2}^{\prime \prime}\right)}$ choices.) So

$$
\bar{\Delta}^{\prime}=\overline{\Omega_{0} \oplus\left(\Lambda_{1} \oplus \Lambda_{1}^{\prime}\right) \oplus\left(\Lambda_{2} \oplus \Lambda_{2}^{\prime} \oplus p \Lambda_{2}^{\prime \prime}\right)}
$$

where $\bar{\Delta}_{1}=\bar{\Lambda}_{1} \oplus \bar{\Lambda}_{1}^{\prime}, \bar{\Delta}_{2}=\overline{\Lambda_{2} \oplus \Lambda_{2}^{\prime}}$.

Now let

$$
\begin{aligned}
\Lambda & =\text { preimage in } \bar{\Delta}^{\prime} \text { of } \overline{\Omega_{0} \oplus \Lambda_{1} \oplus \Lambda_{2}} \\
& =\Omega_{0} \oplus\left(\Lambda_{1} \oplus p \Lambda_{1}^{\prime}\right) \oplus\left(\Lambda_{2} \oplus p \Lambda_{2}^{\prime} \oplus p^{2} \Lambda_{2}^{\prime \prime}\right) .
\end{aligned}
$$

As noted above, $\Omega \cap \Lambda / p(\Omega+\Lambda) \simeq \Lambda_{1} / p \Lambda_{1} \oplus p \Lambda_{2}^{\prime} / p^{2} \Lambda_{2}^{\prime}$ and $p \Lambda_{2}^{\prime} / p^{2} \Lambda_{2}^{\prime}$ is totally isotropic over $\mathbb{Z} / p \mathbb{Z}$. Given a dimension $\ell$ subspace $\bar{U}$ in $\Lambda_{1} / p \Lambda_{1}$ with basis $\left\{x_{1}, \ldots, x_{\ell}\right\}$, there are $\beta\left(d_{2}^{\prime}, d-\ell\right) p^{\ell\left(d_{2}^{\prime}-d+\ell\right)}$ dimension $d=d_{1}+d_{2}^{\prime}-n+j$ subspaces of $\Lambda_{1} / p \Lambda_{1} \oplus p \Lambda_{2}^{\prime} / p^{2} \Lambda_{2}^{\prime}$ that project onto $\bar{U}$ in $\Lambda_{1} / p \Lambda_{1}$. So for each $\Lambda, \alpha_{j}(\Omega, \Lambda)$ is the number of $\bar{U}$ constructed above, times $\beta\left(d_{2}^{\prime}, d-\ell\right) p^{\ell\left(d_{2}^{\prime}-d+\ell\right)}$.

We know that $E_{j}(\Omega, \Lambda)=j(k-n-1)+k\left(d_{2}-r_{0}-t\right)+\left(r_{0}+t\right)\left(n-d_{2}+1\right)+d(d+1) / 2$. Also, by several applications of Lemma 5.1 (a), we find that

$$
\beta(r, a) \beta(r-a, b) \beta(r-a-b, c)=\beta(r, b+c) \beta(b+c, b) \beta(r-b-c, a) .
$$

Thus with $r=r_{2}, a=d_{2}^{\prime \prime}, b=d_{2}, c=d-\ell$, and $x=d_{2}+d-\ell=j-r_{0}-\ell-t$ we have $d=j-r_{0}-d_{2}-t$ so

$$
\begin{aligned}
c_{j}^{*}(\Omega) & =p^{j(k-n-1)} \sum_{\ell, d_{2}, t} \varphi_{\ell}\left(\bar{\Omega}_{1}\right) p^{k\left(d_{2}-r_{0}-t\right)+\left(r_{0}+t\right)\left(n-d_{2}+1\right)+d(d+1) / 2+d_{2} t+\ell\left(\ell-d+r_{2}-d_{2}\right)} \\
& \cdot \beta\left(r_{2}, x\right) \beta\left(x, d_{2}\right) \sum_{d_{1}^{\prime}+d_{2}^{\prime \prime}=t} p^{d_{2}^{\prime \prime}\left(r_{1}-d_{1}^{\prime}-\ell\right)} \beta\left(r_{2}-x, d_{2}^{\prime \prime}\right) \beta\left(r_{1}-\ell, d_{1}^{\prime}\right) .
\end{aligned}
$$

By Lemma $5.1(\mathrm{c})$, the sum on $d_{1}^{\prime}+d_{2}^{\prime \prime}=t$ reduces to $\beta\left(r_{1}+r_{2}-x-\ell, t\right)=$ $\beta(n-j+t, t)$. Thus, after substituting for $d$ and simplifying, we have

$$
\begin{array}{r}
c_{j}^{*}(\Omega)=\sum_{\ell, t} \varphi_{\ell}\left(\bar{\Omega}_{1}\right) p^{E} \beta\left(r_{2}, x\right) \beta(n-j+t, t) \\
\cdot \sum_{d_{2}} p^{d_{2}(k-j+t)+d_{2}\left(d_{2}-1\right) / 2} \beta\left(x, d_{2}\right)
\end{array}
$$

where

$$
E=(k-n)\left(j-r_{0}-t\right)+\left(j-r_{0}-t\right)\left(j-r_{0}-t-1\right) / 2+\ell\left(\ell+n-j-r_{1}+t\right) .
$$

By Lemma 5.2 the sum on $d_{2}$ is

$$
\prod_{i=1}^{x}\left(p^{k-r_{0}-\ell-i}+1\right)=\epsilon\left(k-j+t, j-r_{0}-\ell-t\right),
$$


proving the proposition.

Proof of Proposition 3.2. (1) Let $K$ vary over the preimages in $L$ of all maximal, totally isotropic subspaces $\bar{C}$ of $L / p L$. We are assuming $\chi(p)=1$, which means $L / p L \simeq \mathbb{H}^{k}$, and so there are $\varphi_{k}\left(\mathbb{H}^{k}\right)=\epsilon(0, k)$ such $K$. Note that when scaled by $1 / p, K / p K \simeq \mathbb{H}^{k}$. Thus $\mathbb{Z}_{p} K^{1 / p} \simeq \mathbb{Z}_{p} L$, and for $q$ any prime not equal to $p, \mathbb{Z}_{q} K \simeq \mathbb{Z}_{q} L$. (So if for all $q$, either all the modular components of $\mathbb{Z}_{q} L$ are of even rank or $\left(\frac{p}{q}\right)=1$, then $K^{1 / p} \in$ gen $L$. This is necessarily the case if $\chi=1$.) Take $\Omega=\Omega_{1} \oplus p \Omega_{2}, r_{i}=\operatorname{rank} \Omega_{i}, \Omega_{1}$ primitive in $L$ modulo $p, Q(\Omega) \equiv 0(\bmod p)$ (as in Proposition 3.1 (1)). Then $\Omega \subseteq K$ if and only if $\bar{\Omega}_{1} \subseteq \bar{C}$ in $L / p L$. The number of such $\bar{C}$ is the number of ways to extend $\bar{\Omega}_{1}$ to a dimension $k$ totally isotropic subspace of $L / p L$. We know $\bar{\Omega}_{1}$ is totally isotropic of dimension $r_{1}$, so $L / p L=\left(\bar{\Omega}_{1} \oplus \bar{\Delta}_{1}\right) \perp \mathbb{H}^{k-r_{1}}$ where $\bar{\Omega}_{1} \oplus \bar{\Delta}_{1} \simeq \mathbb{H}^{r_{1}}$. Thus the number of ways to extend $\bar{\Omega}_{1}$ to $\bar{C}$ is the number of dimension $k-r_{1}$ totally isotropic subspaces of $\mathbb{H}^{k-r_{1}}$, which is

$$
\epsilon\left(0, k-r_{1}\right)=\epsilon\left(0, k-n+r_{2}\right)=\epsilon(0, k-n) \epsilon\left(k-n, r_{2}\right) .
$$

(2) We now construct all lattices $K_{j}$ such that $p L \subseteq K_{j} \subseteq \frac{1}{p} L$, $\operatorname{mult}_{\left\{L: K_{j}\right\}}(p)=$ mult $_{\left.L: K_{j}\right\}}(1 / p)=j$, and $K_{j} \in$ gen $L$. We count how many $K_{j}$ contain any given integral $\Omega \subseteq \frac{1}{p} L$. Then we compare $\theta(L) \mid \widetilde{T}_{j}\left(p^{2}\right)$ to the sum $\sum_{K_{j}} \theta\left(K_{j}\right)$.

To construct all $K_{j}$, we take a dimension $j$ totally isotropic subspace $\bar{C}$ of $L / p L$, and let $K^{\prime}$ be the preimage in $L$ of $\bar{C}$. The number of choices for $\bar{C}$ is $\varphi_{j}(L / p L)$. Since $L / p L$ is regular, there is some $\bar{D}$ so that $\bar{C} \oplus \bar{D} \simeq\left(\begin{array}{ll}0 & I \\ I & 0\end{array}\right)$ (over $\mathbb{Z} / p \mathbb{Z}$ ). So $\bar{C} \oplus \bar{D}$ is regular and thus splits $L / p L: L / p L=(\bar{C} \oplus \bar{D}) \perp \bar{J}$. So $K^{\prime}=(C \oplus p D) \oplus p J$, and in $K^{\prime} / p K^{\prime}$ (scaled by $\left.1 / p\right), \overline{p J}=\operatorname{rad} K^{\prime} / p K^{\prime}$. Also, $\overline{C \oplus p D} \simeq \mathbb{H}^{j}(\mathbb{H} \simeq$ $\left(\begin{array}{ll}0 & 1 \\ 1 & 0\end{array}\right)$ a hyperbolic plane) and $\overline{p D}$ is totally isotropic. Thus there is a totally isotropic $\overline{C^{\prime}}$ so that $\overline{C^{\prime} \oplus p D}=\overline{C \oplus p D}$.

In fact, the number of such $\overline{C^{\prime}}$ is the number of dimension $j$ totally isotropic sub spaces of $\overline{C \oplus p D}$ (scaled by $1 / p$ ) that are independent of $\overline{p D}$. To count, choose isotropic $\bar{x}_{1} \in \overline{C \oplus p D}, \bar{x}_{1} \notin \overline{p D}$ (we have $\left(p^{j}-1\right)\left(p^{j-1}+1\right)$ choices); then choose any $\bar{y}_{1} \in \overline{p D}$ so that $\left\langle\bar{x}_{1}, \bar{y}_{1}\right\rangle \simeq \mathbb{H}$. (So $\overline{C \oplus p D}=\left\langle\bar{x}_{1}, \bar{y}_{1}\right\rangle \perp W, W \simeq \mathbb{H}^{j-1}$.) Then for $1<i \leq j$, we have $\overline{C \oplus p D}=<\bar{x}_{1}, \bar{y}_{1}, \ldots, \bar{x}_{i-1}, \bar{y}_{i-1}>\perp\left(\overline{C_{i} \oplus D_{i}}\right)$, $<\bar{x}_{1}, \bar{y}_{1}, \ldots, \bar{x}_{i-1}, \bar{y}_{i-1}>\simeq \mathbb{H}^{i-1}, \bar{C}_{i} \subseteq \bar{D}$, and $\overline{p D_{i}} \subseteq \overline{p D}$. Choose isotropic $\bar{x}_{i} \in$ $\left\langle\bar{x}_{1}, \ldots, \bar{x}_{i-1}\right\rangle^{\perp}=\bar{x}_{i} \in\left\langle\bar{x}_{1}, \ldots, \bar{x}_{i-1}\right\rangle \perp\left(\bar{C}_{i} \oplus \overline{p D_{i}}\right), \bar{x}_{i} \notin\left\langle\bar{x}_{1}, \ldots, \bar{x}_{i-1}\right\rangle \oplus \overline{p D_{i}}$, and choose $\bar{y}_{i} \in \overline{p D}, \bar{y}_{i} \notin\left\langle\bar{y}_{1}, \ldots, \bar{y}_{i-1}\right\rangle$, so that $\left\langle\bar{x}_{i}, \bar{y}_{i}\right\rangle \simeq \mathbb{H}$. When counting the number of $\overline{C^{\prime}}=\left\langle\bar{x}_{1}, \ldots, \bar{x}_{j}\right\rangle$, the choices of $\bar{y}_{i}$ are inconsequential, and we find that the number of (ordered) bases $\bar{x}_{1}, \ldots, \bar{x}_{j}$ we construct is

$$
\prod_{i=0}^{j-1} p^{i}\left(p^{j-i}-1\right)\left(p^{j-i-1}+1\right)-p^{i}\left(p^{j-i}-1\right) .
$$


The number of $\overline{C^{\prime}}$ is this divided by $\prod_{i=0}^{j-1} p^{i}\left(p^{j-i}-1\right)$, the number of bases for any given $\overline{C^{\prime}}$. Thus the number of $\overline{C^{\prime}}$ is $p^{j(j-1) / 2}$.

Let $p K_{j}$ be the preimage in $K^{\prime}$ of ${\overline{C^{\prime}}}^{\perp}=\overline{C^{\prime} \oplus p J}$. Thus $L=C^{\prime} \oplus D \oplus J$ and $K_{j}=\frac{1}{p} C^{\prime} \oplus p D \oplus J$; since $\overline{C^{\prime}}$ is totally isotropic in $K^{\prime} / p K^{\prime}$ (which is scaled by $1 / p)$, we have $Q\left(C^{\prime}\right) \equiv 0\left(\bmod p^{2}\right)$. Also, in $L / p L$ we have $B(\bar{C}, \bar{J})=0$, so $B(C, J) \equiv 0(\bmod p)$. Hence $K_{j}$ is integral.

Now fix $K_{j}$ and, as in the preceeding section, fix integral $\Omega \subset \frac{1}{p} L$, and decompose $\Omega$ as $\frac{1}{p} \Omega_{0} \oplus \Omega_{1} \oplus p \Omega_{2}, \Omega_{0}, \Omega_{1}, \Omega_{2} \subseteq L$, with $\Omega_{0}, \Omega_{1}$ primitive in $L$ modulo $p$. We want to determine when $\Omega \subseteq K_{j}$. First note that to have $\Omega \subseteq K_{j}$, we need $\bar{\Omega}_{0} \subseteq \bar{C}$ in $L / p L$; when this holds, $\bar{\Omega}_{0}$ must extend to $\bar{C}^{\prime}$ since $\bar{\Omega}_{0}$ is totally isotropic in $K^{\prime} / p K^{\prime}$. Also, for $x \in L$, we have $x \in K_{j}$ if and only if $x \notin D+p L$; so $x \in K_{j}$ if and only if in $L / p L, \bar{x} \in \overline{C \oplus J}=\bar{C}^{\perp}$. Consequently $\Omega \subseteq K_{j}$ if and only if in $L / p L, \bar{\Omega}_{0} \subseteq \bar{C} \subseteq \bar{\Omega}_{1}^{\perp}$ and in $K^{\prime} / p K^{\prime}, \bar{\Omega}_{0} \subseteq \bar{C}^{\prime}$.

Now we count the number of $K_{j}$ containing a given $\Omega$. Counting the number of ways to extend $\bar{\Omega}_{0}$ to $\overline{C^{\prime}}$ in $K^{\prime} / p K^{\prime}$ is essentially identical to counting the $\overline{C^{\prime}}$, giving us $p^{\left(j-r_{0}\right)\left(j-r_{0}-1\right) / 2}$ choices. Thus we are left with counting the number of ways to extend $\bar{\Omega}_{0}$ to a dimension $j$ totally isotropic subspace $\bar{C} \subseteq \bar{\Omega}_{1}^{\perp}$ in $L / p L$. First observe that $L / p L=\left(\overline{\Omega_{0} \oplus \Delta_{0}}\right) \perp \bar{J}$ for some totally isotropic subspace $\Delta_{0}$ with $\overline{\Omega_{0} \oplus \Delta_{0}} \simeq \mathbb{H}^{r_{0}}\left(r_{0}=\operatorname{dim} \bar{\Omega}_{0}\right)$. Since $L / p L$ is regular, so is $\bar{J}$, and since $\bar{\Omega}_{1} \subseteq \bar{\Omega}_{0}^{\perp}=\overline{C \oplus J}$ with $\bar{\Omega}_{1}$ independent of $\bar{\Omega}_{0}$, we can choose $\bar{\Delta}_{0}$ and $\bar{J}$ so that $\bar{\Omega}_{1} \subseteq \bar{J}$. So extending $\bar{\Omega}_{0}$ to $\bar{C} \subseteq \bar{\Omega}_{1}^{\perp}$ is equivalent to choosing a dimension $j-r_{0}$ totally isotropic subspace of $\bar{\Omega}_{1}^{\perp} \cap \bar{J}$. Let $\bar{R}=\operatorname{rad}\left(\bar{\Omega}_{1}^{\perp} \cap \bar{J}\right.$; thus $\bar{\Omega}_{1}^{\perp} \cap \bar{J}=\bar{R} \perp \bar{U}$ where $\bar{U}$ is regular. Since $\bar{J}$ is regular, we have $\bar{J}=\left(\bar{R} \oplus \bar{R}^{\prime}\right) \perp \bar{U} \perp \bar{U}^{\prime}$ where $\bar{R} \oplus \bar{R}^{\prime} \simeq \mathbb{H}^{s}$ where $s=\operatorname{dim} \bar{R}$, and $\bar{U}^{\prime}$ is regular. Also, $\bar{\Omega}_{1}^{\perp} \cap \bar{J}=\bar{R} \perp \bar{U}^{\prime}$, and this has dimension $2\left(k-r_{0}\right)-r_{1}$. We know $\bar{U} \perp \bar{U}^{\prime}$ is regular, and it is hyperbolic exactly when $L / p L$ is. Thus if $\bar{U}$ is hyperbolic, $\bar{U}^{\prime}$ is hyperbolic exactly when $L / p L$ is. Recalling that the number of dimension $\ell$ totally isotropic subspaces of an odd dimensional, regular space depends only on its dimension, we find

$$
\varphi_{j-r_{0}}\left(\bar{\Omega}_{1}^{\perp} \cap \bar{J}\right)= \begin{cases}\varphi_{j-r_{0}}\left(\bar{\Omega}_{1} \perp \mathbb{H}^{k-r_{0}-r_{1}}\right) & \text { if } L / p L \simeq \mathbb{H}^{k}, \\ \varphi_{j-r_{0}}\left(\bar{\Omega}_{1} \perp \mathbb{H}^{k-r_{0}-r_{1}-1} \perp \mathbb{A}\right. & \text { if } \Lambda / p L \simeq \mathbb{H}^{k-1} \perp \mathbb{A} .\end{cases}
$$

(Recall that we have assumed $n \leq k$, thus $\operatorname{dim} \bar{U}^{\prime} \geq \operatorname{dim} \bar{U}$. Also, here $\mathbb{A}$ denotes an anisotropic plane, i.e. $\mathbb{A} \simeq\langle 1,-\delta\rangle$ where $\left(\frac{\delta}{p}\right)=-1$.) So when $L / p L \simeq \mathbb{H}^{k}$, the number of $K_{j}$ containing $\Omega$ is

$$
b_{j}(\Omega)=p^{\left(j-r_{0}\left(j-r_{0}-1\right) / 2\right.} \varphi_{j-r_{0}}\left(\bar{\Omega}_{1} \perp \mathbb{H}^{k-r_{0}-r_{1}}\right)
$$

and by the Reduction Lemma (Lemma 4.1), 


$$
\begin{aligned}
= & p^{\left(j-r_{0}\left(j-r_{0}-1\right) / 2\right.} \sum_{\ell} \varphi_{\ell}\left(\bar{\Omega}_{1}\right) p^{\ell\left(k-j-r_{1}+\ell\right)} \\
& \cdot \epsilon\left(k-j, j-r_{0}-\ell\right) \beta\left(k-r_{0}-r_{1}, j-r_{0}-\ell\right) .
\end{aligned}
$$

On the other hand,

$$
\begin{aligned}
\sum_{\ell, t} & A_{j}(\ell, t, \Omega) p^{t(t-1) / 2} \beta(k-n, t) \epsilon(k-j, t) \\
= & \sum_{\ell} p^{\left(j-r_{0}\left(j-r_{0}-1\right) / 2+\ell\left(k-j-r_{1}+\ell\right)\right.} \varphi_{\ell}\left(\bar{\Omega}_{1}\right) \epsilon\left(k-j, j-r_{0}-\ell\right) \\
& \cdot \sum_{t} p^{(k-n-t)\left(j-r_{0}-\ell-t\right)} \beta(k-n, t) \beta\left(r_{2}, j-r_{0}-\ell-t\right)
\end{aligned}
$$

and by Lemma 5.1(c)

$$
\begin{aligned}
& =p^{\left(j-r_{0}\left(j-r_{0}-1\right) / 2\right.} \sum_{\ell} \varphi_{\ell}\left(\bar{\Omega}_{1}\right) p^{\ell\left(k-j-r_{1}+\ell\right)} \\
& \cdot \epsilon\left(k-j, j-r_{0}-\ell\right) \beta\left(k-r_{0}-r_{1}, j-r_{0}-\ell\right) \\
& =b_{j}(\Omega) . \quad \square
\end{aligned}
$$

\section{Proof of Lemma 4.1 (The Reduction lemma)}

Say $V$ is isometric to a hyperbolic plane orthogonal to $U$. Take $W$ to be a totally isotropic, dimenstion $\ell$ subspace of $U \perp V$. Then $W=\left\langle u_{1}+v+1, \ldots, u_{\ell}+v_{\ell}>\right.$ where $u_{i} \in U, v_{i} \in V$. Consider $W^{\prime}=<u_{1}, \ldots, u_{\ell}>$ (so $W^{\prime}$ is the projection of $W$ onto $U$ ). We cannot have $u_{1}$ and $u_{2}$ equal to 0 , for this would require $<v_{1}, v_{2}>$ to be totally isotropic of degree 2 . So assume $u_{2} \neq 0$.

We want to examine how many $W$ project to the same $W^{\prime}$. Suppose first that $W^{\prime}=<u_{2}, \ldots, u_{\ell}>$ has dimension $\ell-1$. Then $v_{1}$ must be (nonzero and) isotropic in $V$ with $B\left(v_{1}, v_{i}\right)=B\left(v_{1}, u_{i}+v_{i}\right)=0$ for all $i \geq 2$. Since $<v_{1}>^{\perp}=<v_{1}>$ when $v_{1}$ is isotropic (recall that $V$ is a hyperbolic plane), we must have $v_{i} \in<v_{1}>$ for all $i \geq 2$ and hence

$$
W^{\prime}=<v_{1}, u_{2}, \ldots, u_{\ell}>\text {. }
$$

Since there are 2 isotropic lines $\left\langle v_{1}\right\rangle$ in $V$, there are 2 subspaces $W$ that project onto this $W^{\prime}$. Notice that $\left\langle u_{2}, \ldots, u_{\ell}\right\rangle$ must be totally isotropic.

Now say $W^{\prime}=<u_{1}, u_{2}, \ldots, u_{\ell}>$ is totally isotropic of dimension $\ell$. Then $Q\left(v_{i}\right)=Q\left(u_{i}+v_{i}\right)=0$ for all $i$ and $B\left(v_{i}, v_{j}\right)=B\left(u_{i}+v_{i}, u_{j}+v_{j}\right)=0$. Hence the $v_{i}$ all lie on the same isotropic line. Thus there are $p^{\ell}$ spaces $W$ that project onto this $W^{\prime}$ with $v_{i}$ on a given line; one of these $W$ is $W^{\prime}$. Hence there are $2 p^{\ell}-1$ distinct $W$ that project onto $W^{\prime}$.

Say $W^{\prime}$ has dimension $\ell, W^{\prime} \simeq<\varepsilon>\perp<0, \ldots, 0>$ with $\varepsilon \neq 0$. Then $Q\left(v_{1}\right)=$ $-Q\left(u_{1}\right)=-\varepsilon, Q\left(v_{i}\right)=0$ for all $i \geq 2$, and $B\left(v_{1}, v_{i}\right)=0$ for all $i \geq 2$. There are 
$(p-1) / 2$ anisotropic lines in $V$ that represent squares, and $(p-1) / 2$ that represent non-squares. Each anisotropic line represents a nonzero value either twice or not at all. So there are $p-1$ vectors $v_{1} \in V$ with $Q\left(v_{1}\right)=-\varepsilon$. For such $v_{1},\left\langle v_{1}\right\rangle^{\perp}$ is anisotropic, so $v_{2}, \ldots, v_{\ell}=0$. Thus there are $p-1$ dimension $\ell$, totally isotropic spaces $W$ that project onto $W^{\prime}$.

Finally, say $W^{\prime}$ has dimension $\ell$ with $W^{\prime} \simeq\left(\begin{array}{ll}0 & 1 \\ 1 & 0\end{array}\right) \perp<0, \ldots, 0>$. Then $Q\left(v_{1}\right)=Q\left(u_{1}+v_{1}\right)=0=Q\left(u_{2}+v_{2}\right)=Q\left(v_{2}\right)=0, B\left(v_{1}, v_{2}\right)=-B\left(u_{1}, u_{2}\right)=-1$, $B\left(v_{1}, v_{i}\right)=B\left(v_{2}, v_{i}\right)=0$ for all $i \geq 3$. So $v_{1}, v_{2}$ span $V$, and $v_{3}, \ldots, v_{\ell} \in<$ $v_{1}, v_{2}>^{\perp}=\{0\}$. Given $u_{1}, u_{2}$, there are $2(p-1)$ isotropic $v_{1}$ in $V$; having chosen $v_{1}$, there is exactly one isotropic $v_{2} \in V$ so that $B\left(v_{1}, v_{2}\right)=-1$. Thus ther are $2(p-1)$ spaces $W$ that project onto $W^{\prime}$.

We can classify all dimension $\ell$, totally isotropic $W$ in $U \perp V$ by their projections onto $U$. We have:

$$
\begin{aligned}
\varphi_{\ell}(U \perp \mathbb{H})=2 & \cdot \#\left\{\operatorname{dim} \ell-1, \text { totally isotropic } W^{\prime} \subseteq U\right\} \\
& +\left(2 p^{\ell}-1\right) \cdot \#\left\{\operatorname{dim} \ell, \text { totally isotropic } W^{\prime} \subseteq U\right\} \\
& +(p-1) \cdot \#\left\{\operatorname{dim} \ell W^{\prime} \subseteq U, W^{\prime} \simeq<\varepsilon>\perp<0, \ldots, 0>\right\} \\
& +2(p-1) \cdot\left\{\operatorname{dim} \ell W^{\prime} \subseteq U, W^{\prime} \simeq \mathbb{H} \perp<0, \ldots, 0>\right\} .
\end{aligned}
$$

On the other hand, we can take each dimension $\ell-1$ totally isotropic $U^{\prime} \subseteq U$ and extend to a dimension $\ell$ subspace $W^{\prime} \subseteq U$. We get:

$$
\begin{aligned}
\varphi_{\ell-1}(U) \beta(\operatorname{dim} U+1-\ell, 1) \\
=\#\left\{\left(U^{\prime}, W^{\prime}\right): U^{\prime}, W^{\prime} \subseteq U, \operatorname{dim} U^{\prime}=\ell-1, \operatorname{dim} W^{\prime}=\ell, U^{\prime} \text { totally isotropic }\right\} \\
=\#\left\{\operatorname{dim} \ell, \text { totally isotropic } W^{\prime} \subseteq U\right\} \beta(\ell, \ell-1) \\
\quad+\#\left\{\operatorname{dim} \ell W^{\prime} \subseteq U, W^{\prime} \simeq<\varepsilon>\perp<0, \ldots, 0>\right\} \\
\quad+\#\left\{\operatorname{dim} \ell W^{\prime} \subseteq U, W^{\prime} \simeq \mathbb{H} \perp<0, \ldots, 0>\right\} \cdot 2 .
\end{aligned}
$$

Therefore

$$
\begin{aligned}
\varphi_{\ell}(U \perp \mathbb{H})-(p-1) \varphi_{\ell-1}(U) \beta(\operatorname{dim} U+1-\ell, 1) \\
=2 \varphi_{\ell-1}(U)+p^{\ell} \varphi_{\ell}(U),
\end{aligned}
$$

that is, $\varphi_{\ell}(U \perp \mathbb{H})=\left(p^{\operatorname{dim} U+1-\ell}+1\right) \varphi_{\ell-1}(U)+p^{\ell} \varphi_{\ell}(U)$.

This shows that the proposition holds for $t=1$. Now we argue by induction on $t$. We know the identity holds for all $\ell$ when $t=1$. Take $t>1$. Using the induction hypothesis and our formula for $\varphi_{*}(U \perp \mathbb{H})$, we get

$$
\begin{aligned}
\varphi_{\ell}\left(U \perp \mathbb{H}^{t}\right)= & \varphi_{\ell}\left((U \perp \mathbb{H}) \perp \mathbb{H}^{t-1}\right) \\
= & \sum_{0 \leq r \leq t-1} p^{(t-1-r)(\ell-r)} \epsilon(d+1+t-\ell, r) \beta(t-1, r) \\
& \cdot\left(\epsilon(d+1+r-\ell, 1) \varphi_{\ell-r-1}(U)+p^{\ell-r} \varphi_{\ell-r}(U)\right) .
\end{aligned}
$$


Split this into two sums on $r$, and in the first replace $r$ by $r-1$. Then we have a linear combination of the $\varphi_{\ell-r}(U)$; the coefficient on $\varphi_{\ell}(U)$ is $p^{t \ell}$, the coefficient on $\varphi_{\ell-t}(U)$ is $\epsilon(d+t-\ell, t)$, and for $0<r<t$, the coefficient on $\varphi_{\ell-r}(U)$ is

$$
\begin{gathered}
p^{(t-r)(\ell-r)} \frac{\beta(t-1, r-1)}{\left(p^{r}-1\right)} \epsilon(d+t+1-\ell, r-1) \\
\cdot\left[p^{t-r}\left(p^{r}-1\right)\left(p^{d+r-\ell}+1\right)+\left(p^{t-r}-1\right)\left(p^{d+t+r-\ell}+1\right)\right] \\
=p^{(t-r)(\ell-r)} \beta(t, r) \epsilon(d+t-\ell, r) .
\end{gathered}
$$

The lemma now follows.

\section{Supplementary Lemmas}

Lemma 5.1.

(a) $\beta(r, m) \beta\left(r-m, m^{\prime}\right)=\beta\left(r, m^{\prime}\right) \beta\left(r-m^{\prime}, m\right)$.

(b) $\beta(r, m) \beta\left(r-m, m^{\prime}\right)=\beta\left(r, m+m^{\prime}\right) \beta\left(m+m^{\prime}, m\right)$.

(c) $\sum_{0 \leq d \leq r} p^{d(r-t+d)} \beta(r, t-d) \beta\left(r^{\prime}, d\right)=\beta\left(r+r^{\prime}, t\right)$.

(d) $\beta\left(r-m+1, m^{\prime}+1\right) \beta(r+1, m)-\beta\left(m+m^{\prime}, m^{\prime}\right) \beta\left(r+1, m+m^{\prime}+1\right)=$ $p^{m^{\prime}+1} \beta\left(m+m^{\prime}, m^{\prime}+1\right) \beta\left(r+1, m+m^{\prime}+1\right)$.

Proof. (a) Use that

$$
\beta(r, m)=\prod_{i=0}^{m-1} \frac{p^{r-i}-1}{p^{m-i}-1} .
$$

(b) Choose an $m+m^{\prime}$-dimensional subspace of an $r$-dimensional space by first choosing an $m$-dimensional subspace, then extending that to a $m+m^{\prime}$-dimensional space. Thus each $m+m^{\prime}$-dimensional subspace is constructed $\beta\left(m+m^{\prime}, m\right)=$ $\beta\left(m+m^{\prime}, m^{\prime}\right)$ times, proving identity (b).

(c) Let $r=\operatorname{dim} V, r^{\prime}=\operatorname{dim} V^{\prime}$. We construct all dimension $t$ subspaces of $V \oplus V^{\prime}$ by first choosing a dimension $t-d$ subspace $J$ of $V$ (we have $\beta(r, t-d$ ) choices for each $d, 0 \leq d \leq t$ ), then we extend $J$ to $J \oplus J^{\prime}$ where $\operatorname{dim} J^{\prime}=d$ and $J^{\prime}$ is independent of $V$ (we have $p^{d(r-t+d)} \beta\left(r^{\prime}, d\right)$ choices.

(d) Begin with the identity from (b). Thus

$$
\begin{array}{r}
\beta\left(r-m+1, m^{\prime}+1\right) \beta(r+1, m)-\beta\left(m+m^{\prime}, m^{\prime}\right) \beta\left(r+1, m+m^{\prime}+1\right) \\
=\beta\left(r+1, m+m^{\prime}+1\right)\left[\beta\left(m+m^{\prime}+1, m^{\prime}+1\right)-\beta\left(m+m^{\prime}, m^{\prime}\right)\right] .
\end{array}
$$

Using the formula for $\beta(*, *)$ (or arguing combinatorially), one easily verifies that $\beta\left(m+m^{\prime}+1, m^{\prime}+1\right)-\beta\left(m+m^{\prime}, m^{\prime}\right)=p^{m^{\prime}+1} \beta\left(m+m^{\prime}, m^{\prime}+1\right)$. Substituting this value in the previous equation yields the result. 


\section{Lemma 5.2.}

$$
\sum_{0 \leq d \leq m} p^{d(y-m)+d(d-1) / 2} \beta(m, d)=\prod_{i=1}^{m}\left(p^{y-i}+1\right)=\epsilon(y-m, m) .
$$

Proof. We argue by induction on $m$, first noting that the identity trivially holds for $m=0,1$. Take $m \geq 1$. Then

$$
\begin{aligned}
& \sum_{0 \leq d \leq m+1} p^{d(d-m-1)+d(d-1) / 2} \beta(m+1, d) \\
&=1+p^{(m+1)(y-m-1)+m(m+1) / 2} \\
&+\sum_{1 \leq d \leq m} p^{d(y-m-1)+d(d-1) / 2}\left(\beta(m, d)+p^{m-d} \beta(m, d-1)\right)
\end{aligned}
$$

by (c) of Lemma 5.1 and with $y^{\prime}=y-1$,

$$
=\left(p^{y^{\prime}}+1\right) \sum_{0 \leq d \leq m} p^{d\left(y^{\prime}-m\right)+d(d-1) / 2} \beta(m, d)
$$

and by the induction hypothesis

$$
\begin{aligned}
& =\left(p^{y^{\prime}}+1\right) \prod_{i=1}^{m}\left(p^{y^{\prime}-i}+1\right) \\
& =\prod_{i=1}^{m+1}\left(p^{y-i}+1\right) \cdot \square
\end{aligned}
$$

\section{REFERENCES}

1. A.N. Andrianov, Quadratic Forms and Hecke Operators, Grundlehren Math. Wiss., Vol. 286, Springer-Verlag, 1987.

2. E. Artin, Geometric Algebra, Wiley Interscience, 1988.

3. E. Freitag, Siegelsche Modulfunktionen, Grundlehren Math. Wiss., Vol. 254, Springer-Verlag, 1983.

4. E. Freitag, Die Wirkung von Heckeoperatoren auf Thetareihen mit harmonischen Koeffizienten, Math. Ann. 258 (1982), 419-440.

5. J.L. Hafner, L.H. Walling, Explicit action of Hecke operators on Siegel modular forms, J. Number Theory 93 (2002), 34-57.

6. H. Maass, Die Primzahlen in der Theorie der Siegelschen Modulformen, Math. Ann. 124 (1951), 87-122.

7. O.T. O'Meara, Introduction to Quadratic Forms, Springer-Verlag, New York, 1973.

8. S. Rallis, The Eichler commutation relation and the continuous spectrum of the Weil representation, Non-Commutative Harmonic Analysis, Lecture Notes in Mathematics No. 728, Springer-Verlag, 1979, pp. 211-244.

9. S. Rallis, Langlands' functoriality and the Weil represenation, Amer. J. Math 104 (1982), 469-515. 
10. L.H. Walling, Hecke operators on theta series attached to lattices of arbitrary rank, Acta Arith. LIV (1990), 213-240.

11. H. Yoshida, Siegel's modular forms and the arithmetic of quadratic forms, Inv. Math. 60 (1980), 193-248.

12. H. Yoshida, The action of Hecke operators on theta series, Algebraic and topological theories, 1986, pp. 197-238.

L.H. Walling, Department of Mathematics, University of Colorado, Boulder CO 80309 